\title{
Digital Dermatology: Experience From Scotland During Lockdown and Beyond
}

Shareen Muthiah, MBBS; Colin A Morton, MBChB, MD

Department of Dermatology, Stirling Community Hospital, Stirling, United Kingdom

Corresponding Author:

Shareen Muthiah, MBBS

Department of Dermatology

Stirling Community Hospital

Livilands

Stirling, FK8 2AU

United Kingdom

Phone: 4401786434098

Email: shareen.muthiah@nhs.scot

\begin{abstract}
Background: In Scotland, dermatology outpatient services deliver over 300,000 appointments each year. With a significant growth in both new and return attendances, there is an increasing drive for innovative transformation. In response to this challenge, a Digital Dermatology Asynchronous (DDA) consultation platform was co-developed with two National Health Service Dermatology teams. Roll-out of the platform was accelerated during Scotland's initial COVID-19 lockdown and its wider scope was prospectively evaluated.
\end{abstract}

Objective: The aims of the platform were to (1) improve the patient experience by reducing the need to attend hospital for consultations; (2) modernize delivery of outpatient care, providing clinicians with a store-and-forward form of telemedicine; (3) use an integrated digital platform-linked with booking systems and the electronic patient records-to increase efficiency and capacity, thereby creating a more sustainable outpatient service; and (4) create a positive environmental impact by reducing travel and hence the carbon footprint.

Methods: During an 11-week "lockdown" period from late March 2020, a total of 405 consultations were prospectively audited. Clinicians were asked to complete data collection proformas for each consultation detailing patient demographics, quality of images, diagnosis, and outcomes. The time taken to complete each virtual consultation was recorded for 312 consultations. Feedback surveys were completed by patients and clinicians via email.

Results: Of the 405 consultations, 297 new and 108 returning patient consultations were assessed, with $80 \%$ of submitted images being of satisfactory quality. In total, 292 consultations involved the assessment of lesions, with most referred as suspected cancers. Patients of all ages participated, with $31 \%$ of them being aged over 60 years and the parents of 12 children. The consultations were, on average, 3 minutes shorter than equivalent face-to-face (F2F) interactions, and a total of $5758 \mathrm{~km}$ of patient travel was avoided. Outcomes included virtual review (16\%), F2F review (47\%), direct to surgery (11\%), discharge (22\%), and other treatment or investigation (4\%). The majority of those needing F2F review were scheduled for routine follow-up. Patient satisfaction was high, with $82 \%$ of respondents reporting ease of use.

Conclusions: The COVID-19 pandemic has resulted in a paradigm shift in the way we deliver outpatient care. DDA consultations are now operational in 4 health boards and have been successfully included in the choice of consultation type available for patients, helping to augment service capacity during pandemic recovery. The platform is the first of its kind in Scotland, to be integrated with the hospital booking system and electronic patient record and offering a valuable alternative to F2F, telephone, and video consultations.

Conflicts of Interest: None declared.

(iproc 2022;8(1):e36884) doi: $10.2196 / 36884$

\section{KEYWORDS}

outpatient; digital dermatology; consultation survey 


\section{Multimedia Appendix 1}

Patient interface. The platform, which operates through a web-based app, gives patients a 5-day window to submit information and photos of their condition. An assigned clinician responds directly to the patient within an agreed timeframe, and a summary PDF of the consultation is automatically forwarded to their GP.

[PNG File, 1483 KB-Multimedia Appendix 1]

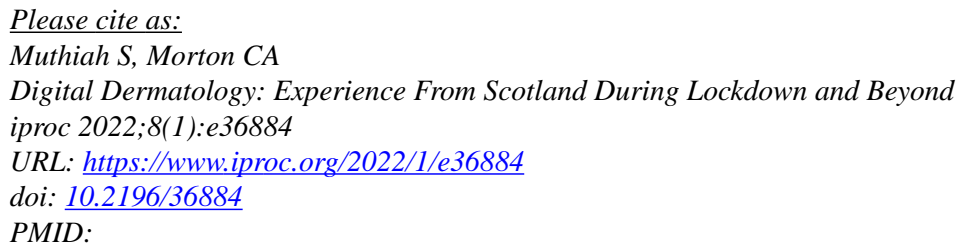

CShareen Muthiah, Colin A Morton. Originally published in Iproceedings (https://www.iproc.org), 25.02.2022. This is an open-access article distributed under the terms of the Creative Commons Attribution License (https://creativecommons.org/licenses/by/4.0/), which permits unrestricted use, distribution, and reproduction in any medium, provided the original work, first published in Iproceedings, is properly cited. The complete bibliographic information, a link to the original publication on https://www.iproc.org/, as well as this copyright and license information must be included. 